\title{
Estrategias de compensación en adultos mayores: Diferencias sociodemográficas y en función de la reserva cognitiva
}

\author{
Teresa Mayordomo ${ }^{1}$, Alicia Sales ${ }^{2}$ y Juan C. Meléndez ${ }^{1}$ \\ ${ }^{1}$ Departamento de Psicología Evolutiva y de la Educación, Facultad de Psicología, Universidad de Valencia \\ ${ }^{2}$ Departamento de Metodología de las Ciencias del Comportamiento, Facultad de Psicología, Universidad de Valencia
}

\begin{abstract}
Resumen: Los términos de reserva cognitiva y compensación, han sido utilizados en una amplia variedad de situaciones en la literatura científica para referirse a la superación o reducción de las pérdidas o déficits en diferentes mecanismos y procesos como es la memoria. A través del Memory Compensation Questionnaire (MCQ), se pretende analizar los diferentes mecanismos de compensación en relación con ciertas variables sociodemográficas (edad, genero y nivel educativo) de una muestra española y establecer, a partir de una medida combinada de reserva cognitiva (RC), las diferencias existentes entre sujetos con alta y baja reserva en el uso de mecanismos de compensación. Se evaluó a 264 sujetos jubilados españoles, donde se obtuvo que las estrategias de compensación más empleadas se basan en los mecanismos de remediación y sustitución. Las estrategias externas y de confianza son las que principalmente muestran correlaciones y diferencias significativas con las diferentes variables sociodemográficas analizadas. En cuanto a la reserva cognitiva, se observó que aquellos sujetos con alta RC presentaban mayor uso de estrategias externo e interno, y los sujetos con baja RC esfuerzo y cambio.
\end{abstract}

Palabras clave: Reserva cognitiva; compensación; envejecimiento; MCQ.
Title: Compensation strategies in older adults: Sociodemographic and cognitive reserve differences.

Abstract: The terms of cognitive reserve and compensation, have been used in a wide variety of situations in the scientific literature to refer to overcome or reduce losses or deficits in different mechanisms and processes such as memory. Through the Memory Compensation Questionnaire (MCQ), is intended to analyze the different mechanisms of compensation in relation to certain sociodemographic variables (age, gender and educational level) from a Spanish sample and establish, from a combined measure of cognitive reserve (CR), the differences between subjects with high and low reserve on using compensation mechanisms. 264 Spanish retirees were assessed. The results showed that the compensation strategies used more frequently were based on remediation and substitution mechanisms. External strategies and confidence are those that show main differences and significant correlations with sociodemographic variables. Regarding cognitive reserve, it was observed that those subjects with higher CR obtained greater use of external strategies and internal, and subjects with lower RC in effort and change. Key words: Cognitive reserve; compensation; aging; MCQ.

\section{Introducción}

El envejecimiento, como etapa del desarrollo, está marcado por importantes cambios de entre los cuales, destacan los relacionados con el funcionamiento cognitivo. Concretamente, y asociado a la edad, existe una predecible pérdida de la eficacia de la memoria en la realización de algunas tareas complejas; si bien, esta pérdida no siempre se produce, siendo tal y como señalan Dixon y Frias (2004), el concepto de compensación uno de los posibles mecanismos que pueden explicar la adaptación cognitiva que desarrollan los sujetos para mantener su eficacia. Por otra parte, y a partir de estudios cerebrales postmortem, se ha podido observar que, sujetos que estructuralmente deberían haber desarrollado una demencia, no manifestaron sus síntomas cognitivoconductuales, surgiendo la denominada hipótesis de la reserva cognitiva (Stern, 2002, 2009), que diferencia entre sujetos con alta y baja reserva cognitiva (ARC y BRC); siendo ambos conceptos importantes variables que pueden minimizar las pérdidas en las funciones cognitivas tales como la memoria. El estudio de las diferencias en el uso de las estrategias de compensación en función de la reserva cognitiva ofrece información valiosa en el análisis diferencial de los grupos propuestos desde esta hipótesis.

La compensación se ha planteado como un intento de maximizar el rendimiento frente al deterioro cognitivo mediante el uso de estrategias implícitas o explícitas. Las estra-

* Dirección para correspondencia [Correspondence address]: Dr. Juan Carlos Meléndez Moral, Dpto. de Psicología Evolutiva y de la Educación, Facultad de Psicología, Universidad de Valencia, Av. Blasco Ibáñez, 21, 46010, Valencia (España).E-mail: melendez@uv.es tegias implícitas, hacen referencia a la modificación de estructuras cerebrales o redes que se ponen en marcha frente al daño cerebral; mientras que las explícitas, son entendidas como un proceso de reorganización en el que el deterioro es compensado por medio de la adquisición o la puesta en marcha de estrategias alternativas de carácter conductual y cognitivo (Díaz-Orueta, Buiza y Yanguas, 2010). De este modo, se pueden diferenciar dos tipos de compensación, una con una perspectiva de carácter más neurológico que plantea un modelo de compensación cerebral, y otra con una perspectiva más psicológica en la que se hace referencia a la compensación a partir de estrategias de tipo comportamental y cognitivo.

Por lo que respecta a las explícitas, los trabajos desarrollados por Frías, Dixon y Bäckman (2003), señalan que cuando hacemos referencia expresa a la memoria, compensar implica el uso de estrategias o mecanismos que ayudan a superar o mitigar la disminución del funcionamiento de ésta, integrando los principales componentes que se pueden identificar para la reducción de los déficits y su compensación en cuatro tipos de actuaciones compensatorias: la remediación (investigar más tiempo en algo para compensar), la sustitución (desarrollar o poner en marcha herramientas latentes que compensen), la acomodación (ajuste de metas y criterio de forma más consonantes con las capacidades) y la asimilación (modificar las demandas o requisitos exigidos por el entorno). De este modo, y a partir de éstas, desarrollan uno de los instrumentos más utilizados para su análisis, el Memory Compensation Questionnaire (MCQ), (Dixon, Frias y Bäckman, 2001), cuya estructura factorial ha sido confirmada en diversas poblaciones (Frias y Dixon, 2005; Van der Elst, Hoo- 
genhout, Dixon, De Groot y Jolles, 2011; Meléndez, Mayordomo, Sales, Cantero y Víguer, 2013).

Entre los principales resultados obtenidos a partir del MCQ, se ha señalado que la utilización de mecanismos de compensación parece ser estable en el tiempo, siempre y cuando se mantenga un nivel cognitivo con un rendimiento y eficacia adecuados. De esta manera, no se percibe la necesidad de aumentar la utilización de conductas compensatorias a fin de mantener niveles anteriores de competencia en situaciones de la vida cotidiana o, ante las exigencias ambientales que se esperan de ellos y sus habilidades y capacidades personales (Bäckman y Dixon, 1992).

Por lo que respecta a la frecuencia de uso, Dixon, Frias y Bäckman (2001) señalan que los mecanismos que más se utilizan, en función de la edad y el género, son los mecanismos de sustitución y remediación; este mayor uso indica que los adultos mayores son conscientes de los beneficios de invertir tiempo en la práctica y de la ampliación de los esfuerzos para recordar información nueva. Ambas estrategias son de carácter interno, principalmente destinadas a la adquisición (codificación) de nueva información; sin embargo, el uso de ayudas de memoria externa (agendas), es la forma más común de la compensación, convirtiéndose según Wilson (1999) en la más eficaz para la rehabilitación de la memoria, con la ventaja de que puede ser adaptada de forma espontánea a múltiples situaciones, aspecto que también se ha comprobado con sujetos con memoria deteriorada. Por otra parte, la estrategia de compensación menos frecuente, es el apoyo en otras personas: cónyuges, amigos, cuidadores, etc., si bien, se ha observado un aumento de su frecuencia en los hombres según aumenta la edad, posiblemente debido a la mayor vulnerabilidad cognitiva.

Por lo que respecta al análisis de variables sociodemográficas, el trabajo de Van der Elst, Hoogenhout, Dixon, De Groot y Jolles (2011), al estudiar el efecto de la edad, el género y el nivel educativo sobre las escalas del MCQ, señala que en los modelos de regresión finales se observó que la edad afecta a las escalas externa, tiempo, esfuerzo, y éxito positivamente. Además, las mujeres obtienen puntuaciones superiores en la escala externo y esfuerzo, y los hombres presentan mayores puntuaciones de la escala de confianza, al igual que los sujetos con menores niveles académicos; por último, este trabajo señala que las puntuaciones de la escala interna y cambio no están significativamente afectadas por ninguna de las variables demográficas. Por otra parte, Dixon, Frias y Bäckman (2001), al estudiar con un intervalo de tres años los patrones de cambio longitudinal en función de la edad y el género para las cinco escalas principales, obtuvieron un efecto principal significativo, tanto en edad, como en género y la interacción de ambos. Señalando que, los efectos significativos de edad entre los hombres se producen en la escala externa y en la de confianza donde además, se observa que los sujetos de más edad presentan un mayor uso de estos mecanismos que los más jóvenes. Además, las mujeres informan de una frecuencia similar de uso de estos mecanismos a través de los grupos de edad. También, se observó que los más mayores y las mujeres informaron de mayor cantidad de éxito, y los más mayores, además presentaron puntuaciones más altas en la escala de cambio. Los resultados del trabajo de Dixon y Frias (2004), cuyo objetivo era examinar los cambios y diferencias interindividuales en un periodo de 6 años, reflejan un aumento en el uso de estrategias flexibles y fáciles de implementar (ayudas externas) y una disminución de las estrategias más exigentes en recursos (estrategias internas mnemotécnicos y de la inversión del tiempo).

Con respecto al concepto de reserva, ésta es entendida como la capacidad que tienen determinadas personas de demorar el posible deterioro cognitivo que acaece con el envejecimiento (Stern, 2009), manteniéndose clínicamente poco afectadas a pesar de que los datos de neuroimagen o neuropatológicos muestren un proceso avanzado de la enfermedad (Rami et al., 2011). Es decir, el sujeto es capaz de soportar mayor cantidad de neuropatología antes de llegar al umbral donde la sintomatología clínica comienza a manifestarse (Lojo-Seoane, Facal, y Juncos-Rabadán, 2012; Manly, Schupf, Tang, Weissm y Stern, 2007; Stern, 2002, 2003). Partiendo de este planteamiento, se han desarrollado dos vías paralelas de investigación, una definida como reserva pasiva, y que depende de características constitucionales del individuo tales como volumen del cerebro, número de neuronas o densidad sináptica (reserva cerebral, Katzman, 1993; reserva neuronal, Mortimer, Schuman y French, 1981); y otra entendida como activa, en la que la conducta actual y previa del sujeto son determinantes, y que queda operacionalizada mediante una medida de reserva cognitiva que permite la diferenciación de sujetos con alta y baja reserva.

Este concepto de reserva cognitiva (RC), fundamentado en variables personales del sujeto, presenta entre sus objetivos el estudio de las variables que podrían estar en la base del efecto protector y que facilitan el uso de la red cerebral de manera más eficiente. Así, la educación, la ocupación, el nivel de inteligencia premórbida, y las actividades de ocio, físicas, mentales y sociales, han sido algunas de las variables más empleadas para la operacionalización de la medida de $\mathrm{RC}$, siendo el análisis factorial exploratorio el procedimiento más empleado para la obtención de una medida combinada a través de éstas variables (Bosch et al., 2010; Corral, Rodríguez, y Amenedo, Sánchez y Díaz, 2006; León, García y Roldán-Tapia, 2011; Lojo-Seoane, Facal y Juncos-Rabadán, 2012; Meléndez, Mayordomo y Sales, 2012; Rami et al., 2011; Solé-Padullés et al., 2009; Stern, 2009; Stern et al., 2005; Valenzuela y Sachdev, 2006a).

Partiendo de este tipo de medidas, la literatura científica ha mostrado la existencia de relación entre el nivel de ejecución cognitivo y la reserva cognitiva, tanto en sujetos sanos como con diferentes patologías cognitivas. El estudio de Corral et al., (2006) con sujetos sanos, señala que aquellos con $\mathrm{BRC}$ obtienen puntuaciones inferiores en atención, CI, memoria y funciones globales, que aquellos con ARC. El estudio de Santamarina y Corral (2009), con sujetos con epilepsia del lóbulo temporal, muestran que los pacientes con BRC 
presentan mayor morbilidad cognitiva, además de un rendimiento neuropsicológico significativamente inferior al del grupo con ARC en atención, memoria, lenguaje y habilidades visuoconstructivas. En el trabajo de Drag y Bieliauskas (2010), los índices de la reserva cognitiva se asocian positivamente con el rendimiento cognitivo de dominios múltiples, incluyendo la atención y la memoria. También, y a partir de las revisiones meta-analíticas de Valenzuela y Sachdev (2006a; 2006b), se concluye que el riesgo de aparición de síntomas de demencia es un $46 \%$ más bajo en aquellos sujetos con alta reserva. De este modo, se puede afirmar que la RC puede ser entendida como un factor protector contra la expresión del deterioro cognitivo relacionado con la edad, permitiendo además un adecuado rendimiento cognitivo cuanta mayor sea la capacidad de reserva. En este sentido, Stern (2006) afirma que dos personas con características clínicas similares pueden tener niveles diferentes de la patología subyacente de la enfermedad de Alzheimer (EA), siendo la RC la que puede explicar parte de esta disparidad.

De este modo, desde el presente trabajo, se plantea como primer objetivo estudiar en una muestra de adultos mayores sanos las puntuaciones de las subescalas del MCQ, su relación con la edad, así como la existencia de posibles diferencias de las subescalas del MCQ con diferentes sociodemográficos. Partiendo de la existencia de un enlentecimiento en las funciones cognitivas asociado a la edad, se espera que aquellos sujetos de mayor edad presenten puntuaciones más elevadas en el uso de las estrategias de compensación. Por lo que respecta al género, se plantea que los hombres obtendrán puntuaciones significativamente superiores en la utilización de la estrategia de confianza, mientras que en las demás estrategias no existirán diferencias. En relación al estado civil, se espera que la utilización de la estrategia de confianza se aplique en mayor medida en aquellos sujetos casados, lo cuales deberían mostrar puntuaciones más elevadas que viudos y solteros, dada la existencia del apoyo proporcionado por la pareja, figura que es esencial en esta etapa del ciclo vital. En cuanto al nivel académico, se prevé que aquellos sujetos con un menor nivel de estudios mostrarán un mayor uso de la estrategia de confianza, así como también se prevé la existencia de una mayor percepción de cambio.

En segundo lugar, se pretende estudiar la relación entre la RC y las subescalas del MCQ, analizando si existen diferencias en el uso de las estrategias en función de presentar alta o baja RC. Para su desarrollo, se parte de que aquellos sujetos con mayor capacidad de reserva, y por tanto con un mayor rendimiento cognitivo, tendrán la oportunidad de obtener un mayor beneficio de éstas capacidades, enriqueciéndose en mayor medida del uso de éstas a través de diversas estrategias cognitivas y conductuales al poseer una mayor gama de recursos y competencias; de este modo, los sujetos podrán afrontar mejor las pérdidas cognitivas que acaecen con la edad al poseer mayor capacidad de compensación frente a las pérdidas. Así, se pretende desarrollar una medida de RC, a partir de la cual poder discriminar entre los sujetos con alta y baja RC y analizar las posibles diferencias en sus estrategias de compensación, esperando obtener, debido a la existencia de mayores recursos disponibles por parte de los sujetos con alta reserva cognitiva (ARC), puntuaciones diferencialmente significativas en las estrategias externas e internas, así como una menor percepción de cambio y esfuerzo, y una mayor percepción de éxito que los sujetos con baja reserva cognitiva (BRC).

\section{Método}

\section{Participantes}

El diseño es transversal y de encuesta. Participaron un total de 264 adultos mayores de la ciudad de Valencia, España. Todos compartían la condición de jubilados mayores de 65 años y no institucionalizados. La media de edad fue de $74,1(D T=6.8)$ con un rango entre $65-90$; el $62.1 \%$ eran mujeres; $58.3 \%$ casados y un $23.7 \%$ viudos. Con respecto al nivel de estudios: un $21.2 \%$ no tenía estudios, un $50 \%$ tenían estudios primarios, un $15.2 \%$ tenía estudios secundarios y el $13.6 \%$ universitarios.

\section{Instrumentos}

Siempre de forma individual, los participantes contestaron en primer lugar, un cuestionario de datos sociodemográficos respondiendo si en los últimos años realizaban o no una serie de 14 actividades de ocio y estimulantes (pasatiempos, leer, escribir, bailar, ejercicio, deporte, manualidades, estudio/talleres, instrumento, voluntariado, cine, juegos de mesa, pertenencia a asociaciones culturales y viajar). A continuación se aplicó el Mini-Mental State Examination (MMSE) de Folstein, Folstein y Hugh (1975) en su versión en español (Lobo, Saz y Marcos, 2002), requiriéndose una puntuación mínima de 23. Teniendo en cuenta la puntuación del MMSE, 16 sujetos fueron excluidos del estudio, la media final fue de $27.6(D T=2.05)$. Además se administró la subprueba de vocabulario de la Escala de Inteligencia Wechsler para AdultosIII (WAIS-III; Wechsler, 2001) para analizar el nivel de inteligencia premórbida.

Finalmente se aplicó el MCQ (Dixon, Frias y Bäckman, 2001) en su adaptación al español (Meléndez, Mayordomo, Sales, Cantero y Viguer, 2013). El cuestionario se compone de cinco escalas principales que representan formas de conductas compensatorias relevantes en el día a día, de una sexta subescala que evalúa el nivel de satisfacción en las funciones de memoria cotidiana así como la disposición de la persona a acomodarse a los cambios en la memoria, y de una séptima subescala que estudia en qué medida se han producido cambios en los últimos años. Siendo el formato de respuesta tipo Likert (1 a 5), y presentando ítems inversos en todas las escalas.

Las cinco escalas principales son: externa, que hace referencia a todo lo concerniente a apoyos externos como notas, calendarios, etc.; interna, que hace referencia al uso de estrategias mnemotécnicas para la promoción de las funciones 
memorísticas; tiempo, que analiza en qué medida las personas investigan más tiempo para la ejecución de ciertas tareas; esfuerzo, que implica el desarrollo de esfuerzos de cara a la ejecución de las tareas de memoria, de manera que el sujeto se concentre más y se esfuerce más duramente; confianza, que hace referencia a en qué medida los sujetos cuentan con otras personas como apoyo para preguntar o hacer que les recuerden elementos concretos. Las dos escalas complementarias son: éxito, la cual refleja en qué medida los sujetos están comprometidos con la obtención de alto nivel de consecución en tareas relacionadas con las funciones de memoria, lo cual puede reflejar motivación hacia la compensación de las pérdidas producidas por la edad; y cambio, la cual refleja la percepción de cambios en el uso de las anteriores estrategias de compensación en los últimos 5-10 años.

\section{Análisis}

Para el análisis de las diferencias entre los grupos se utilizaron pruebas t para muestras independientes y análisis de varianza con pruebas post hoc de Tuckey. Además se aplicaron correlaciones de Pearson. También se realizó análisis factorial exploratorio para la obtención de la medida ponderada de reserva cognitiva. Los análisis estadísticos se realizaron mediante SPSS 19.

\section{Resultados}

En primer lugar se analizó la frecuencia de uso de las estrategias de compensación por parte de los ancianos sanos, observándose que fueron las dimensiones tiempo $(3.89 ; D T=$ 1.01) y externo $(3.42 ; D T=1.14)$ las utilizadas en mayor medida. Por lo que se refiere a las demás estrategias, las medias fueron: esfuerzo $(2.92 ; D T=1.03)$, interno $(2.74 ; D T=$ $.083)$ y confianza $(2.39 ; D T=.97)$. Además, y en relación a las escalas complementarias del MCQ, los sujetos informaron de importantes cambios en el uso de sus estrategias en los últimos años $(3.71 ; D T=.55)$ con un nivel de éxito de $3.52(D T=1.01)$.

Posteriormente, y en relación al primer objetivo, se analizó el funcionamiento de las estrategias de compensación con diferentes variables de carácter sociodemográfico. De este modo, se aplicaron correlaciones entre la edad y las estrategias del MCQ obteniéndose tan sólo una relación significativa $(p<.01)$ y negativa con la subescala externo $(r=$ $.134)$.

A continuación y a partir de las pruebas $t$ para muestras independientes, se observó la existencia de diferencias de género para la subescala de confianza $(t(256)=2.63 ; p=$ .009) siendo la media de los hombres de 2.6 y la de las mujeres de 2.2.

Al estudiar el estado civil, se observó a partir de los ANOVAs que existían diferencias significativas para la subescala confianza $\left(F_{2,256}=4.36 ; p=.014\right)$, observándose en las pruebas post hoc de Tuckey diferencias entre el grupo de casados con una media de 2.5 con el de viudos con una media de $2.2(p=.040)$ y el de solteros con una media de 2 $(p=.039)$.

Por lo que se refiere al nivel de estudios, los ANOVAS mostraron diferencias entre algunas de las subescalas. La subescala externo mostró diferencias significativas $\left(F_{3,257}=\right.$ 2.91; $p=.035)$, observándose mediante la prueba post hoc de Tuckey diferencias entre los grupos de sin estudios y estudios superiores $(p=.045)$ con medias de 3.1 y 3.8 respectivamente. También se obtuvieron diferencias significativas en la subescala confianza $\left(F_{3,257}=3.38 ; p=.019\right)$ observándose en las pruebas post hoc diferencias entre los sujetos del grupo sin estudios y estudios secundarios $(p=.017)$, con medias de 2.7 y 2.1 respectivamente. Por último, se obtuvieron diferencias significativas en la subescala cambio $\left(F_{3,256}=\right.$ 4.97; $p=.002)$, observándose a partir de la prueba post hoc como el grupo de sin estudios, cuya media es la más alta, es significantemente diferente de los grupos de estudios primarios $(p=.043)$, secundarios $(p=.019)$ y superiores $(p=.003)$, con medias de 3.9, 3.7, 3.6 y 3.5 respectivamente.

En cuanto al segundo objetivo, primero se desarrolló una medida combinada de RC, para a partir de ésta establecer las diferencias existentes respecto a las subescalas del MCQ. Para el desarrollo de la medida de RC, se siguieron las pautas establecidas en otros trabajos (Scarmeas, 2007; Scarmeas et al., 2003; Solé-Padullés et al., 2009; Stern, 2009; Stern et al., 2005), donde se plantean diferentes variablesmedida que están relacionadas directamente con la RC. De este modo, se incluyó el WAIS-III, subprueba vocabulario aplicándose los baremos de conversión en función de la edad, con un rango de puntuaciones entre 1 y 19. Una segunda medida, definida como educación-ocupación, fue codificada usando valores ordinales que en el caso de educación fueron: $1=$ no educación formal, $2=$ primaria, $3=$ secundaria, $4=$ universitaria; y en ocupación: $1=$ manual no cualificado, $2=$ manual cualificado, $3=$ no manual cualificado o técnico, $4=$ profesional (título universitario requerido), 5= manager o director. El valor final se obtuvo sumando los valores educativos y ocupacionales (rango 2-9). Como tercera medida se tuvieron en cuenta actividades de ocio que fueran cognitivamente estimulantes (Scarmeas et al., 2003), que implicaran algún tipo de actividad física y que tuviesen en cuenta la participación social o en grupo evaluadas por los sujetos mediante una respuesta dicotómica de si la realizaban o no y estableciéndose un rango de puntuaciones entre 0 y 14.

Para conocer el peso factorial de estas tres medidas, se realizó un análisis factorial exploratorio con método de componentes principales (Solé-Padullés et al., 2009; Stern et al., 2005) obteniendo una varianza explicada del 51.3\%. Las saturaciones factoriales de cada variable fueron multiplicadas por la puntuación de cada sujeto en cada variable obteniéndose mediante su sumatorio una medida combinada de RC. En la Tabla 1 se pueden ver los principales datos de las variables. 
Tabla 1. Medias, desviaciones típicas, saturación factorial y correlación variable factor de las variables incluidas en la medida de RC.

\begin{tabular}{|c|c|c|c|c|}
\hline & Media & $D T$ & $\lambda$ & $r_{\mathrm{v}-\mathrm{f}}$ \\
\hline Actividades & 4.1 & 2.3 & .813 & $.796^{* *}$ \\
\hline Estudio-Ocupación & 4.0 & 1.9 & .769 & $.718^{* *}$ \\
\hline WAIS-III vocabulario & 9.8 & 2.6 & .541 & $.563^{* *}$ \\
\hline
\end{tabular}

La medida de RC obtuvo una media de $11.93(D T=3.4)$ con un rango entre 4.2 y 21.6. A partir de ésta, los sujetos fueron divididos en dos grupos en función del percentil $25 \mathrm{y}$ 75 , estableciéndose como puntos de corte 9.6 y 13.9 respectivamente. El grupo de alta RC está compuesto por 66 sujetos, con una media de $16.62(D T=1.9)$, y el de baja $\mathrm{RC}$ por 65 sujetos, con una media de $7.89(D T=1.1)$.

Finalmente se estudiaron las diferencias en las subescalas del MCQ en función de presentar alta o baja RC, presentando resultados significativos las escalas de externo, interno, esfuerzo y cambio.

Tabla 2. Diferencia de medias entre los grupos de ARC y BRC en las dimensiones del MCQ.

\begin{tabular}{lllll}
\hline & ARC & BRC & $t$ & Sig \\
\hline Externo & 3.80 & 3.00 & 3.97 & .000 \\
Interno & 2.87 & 2.53 & 2.21 & .028 \\
Tiempo & 3.98 & .386 & .684 & .495 \\
Confianza & 2.38 & 2.52 & .781 & .437 \\
Esfuerzo & 2.74 & 3.27 & 2.91 & .004 \\
Éxito & 3.61 & 3.49 & .645 & .520 \\
Cambio & 3.56 & 3.96 & 4.03 & .000 \\
\hline
\end{tabular}

\section{Discusión}

El análisis de las pérdidas cognitivas en el envejecimiento, y de forma más concreta el estudio de los cambios en memoria, ha supuesto uno de los principales objetivos de estudio en la población mayor. En este marco de análisis, desde los últimos años, dos conceptos han surgido con fuerza como variables que pueden minimizar las pérdidas asociadas a la edad, la compensación y la reserva cognitiva. De este modo, el presente trabajo ha pretendido analizar y poner en relación ambas variables en una muestra de personas mayores.

Inicialmente, se analizó el grado en que los sujetos ponen en marcha los diferentes tipos de estrategias de compensación, y al igual que en el trabajo de Dixon et al (2001), destacar que son los mecanismos de remediación y sustitución los más utilizados, sin embargo y a diferencia de este trabajo, el uso de ayudas de memoria de carácter externo fue la forma más común de compensación utilizada, mientras que en este estudio fue el uso de mayor tiempo, seguido de las estrategias externas. Trabajos anteriores, han observado que el uso de ayudas externas es una de las estrategias preferidas por las personas mayores, dado que es un mecanismo efectivo para soportar el enlentecimiento en el rendimiento de la memoria en la vida cotidiana, y que además, es generalizable a través de diversas situaciones de memoria (Cavanaugh y Poon, 1989; Dixon y Hultsch, 1983; Moscovitch, 1982), de hecho, constituye una de las estrategias más eficaces para la rehabilitación de la memoria (Wilson, 1999). Por otra parte, la estrategia compensatoria menos frecuente es la denominada confianza, que implica el apoyo en los demás (cónyuge, amigos, cuidadores, etc.) para la obtención de ayudas en la actividad cotidiana, y aunque el apoyo estratégico en los demás puede ocurrir regularmente en la vida de los adultos normales (Dixon, Gagnon y Crow, 1998), se puede ver como un mecanismo de compensación sólo en los casos en los que el deterioro ya es presente o va en aumento.

Por lo que respecta al primer objetivo, centrado en el estudio de las diferencias en las escalas del MCQ en función de los aspectos sociodemográficos, tal y como señalan Dixon et al (2001), el uso de los mecanismos de compensación en relación a la edad es bastante estable, y el hecho de que los adultos mayores sin deterioro informen de niveles similares del uso de las estrategias compensatorias, implica que incluso los más mayores, todavía no han percibido la necesidad de incrementar el uso de conductas compensatorias con el fin de mantener niveles eficaces de competencia en las situaciones cotidianas de memoria, o aún no se han enfrentado a un grave desequilibrio entre las exigencias ambientales y sus habilidades y capacidades personales. En este sentido, la no obtención de correlaciones rechazaría la hipótesis planteada, según la cual la necesidad del uso de estrategias con la edad sería mayor dado las pérdidas cognitivas asociadas a la edad; no obstante, confirmaría la idea de la estabilidad en el uso de estrategias hasta la aparición de los primeros síntomas del deterioro. En relación al género, se ha observado cómo en la subescala confianza existen diferencias significativas, siendo más alta la media de los hombres; resultado que es coherente con el trabajo de Dixon et al (2001), en el que al analizar la edad y género de forma conjunta obtuvieron diferencias en la subescala confianza. Este efecto observado, en el que los hombres hacen mayor uso de esta estrategia para compensar las dificultades en tareas cognitivas principalmente cotidianas, podría relacionarse con las diferencias encontradas en el uso de las figuras de apoyo psicológico, en las que según el estudio de Melendez et al (2007), los hombres recurren de forma principal a su cónyuge para la obtención de éste. Del mismo modo, ha sido la estrategia confianza la que ha mostrado diferencias significativas en función del estado civil, siendo el grupo de casados el que mayor media obtiene; se ha de tener en cuenta que en relación al apoyo psicológico e instrumental en la vejez, es la familia cercana la figura más importante en su provisión, siendo el cónyuge una de las figuras más importante (Meléndez, Tomás y Navarro, 2007). Por último, y en relación al nivel de estudios, se ha observado cómo aquellos sujetos con menor nivel son los que diferencialmente perciben un mayor cambio en los últimos años, aspecto que quedaría reforzado por el menor uso de estrategias externas, y por el aumento en el uso de la estrategia de confianza, diferenciación que puede implicar la necesidad de un incremento compensatorio con el fin de mantener niveles eficaces de competencia en las si- 
tuaciones cotidianas de memoria para las que el sujeto no es capaz de adaptarse.

Por lo que respecta al segundo objetivo, se ha presentado un procedimiento para el desarrollo de la medida de RC en una muestra de ancianos sanos, fundamentándose en los criterios principalmente utilizados en la literatura: educaciónocupación, actividades e inteligencia premórbida; de entre estos, actividad y estudios-ocupación han sido las que han obtenido mayor saturación factorial y correlación de la variable con el factor, mientras que la estimación de la inteligencia premórbida, aun siendo una variable a tener en cuenta, ha sido la variable que ha obtenido puntuaciones menores, sustentando lo planteado por Stern (2006), según el cual los criterios educación- ocupación, y actividades, son los que muestran tanto de forma individual como de forma conjunta un mayor efecto sobre la medida de RC. Por otra parte, y al comparar sujetos con AyBRC, los resultados obtenidos muestran en los sujetos con ARC un uso significativamente mayor de las estrategias de externo e interno, uso que según Frias y Dixon (2005), está relacionado con la aplicación de mecanismos de sustitución (externo e interno) y por tanto altamente asociado con el nuevo aprendizaje, de manera que tanto el utilizar ayudas externas como el uso de técnicas mnemónicas en el envejecimiento normal, facilita el rendimiento de memoria. Tal y como señala Stern (2002), los individuos con ARC pueden presentar menor deterioro cogni-

\section{Referencias}

Bäckman, L. y Dixon, R. A. (1992). Psychological compensation: A theoretical framework. Psychological Bulletin, 112, 259-283.

Bosch, B., Bartrés-Faz, D., Rami, L., Arenaza-Urquijo, E., Fernández-Espejo, D., Junqué, C., ..., y Molinuevo, J. L. (2010). Cognitive reserve modulates taskinduced activations and deactivations in healthy elders, amnestic mild cognitive impairment and mild Alzheimer's disease. Cortex, 46, 451-461.

Cavanaugh, J. C. y Poon, L. W. (1989). Metamemorial predictors of memory performance in young and older adults. Psychology and Aging, 4, 365-368

Corral, M., Rodriguez, M., Amenedo, E., Sánchez, J. L. y Díaz, F. (2006). Cognitive reserve, age, and neuropsychological performance in healthy participants. Developmental Neuropsychology, 29, 479-491.

Díaz-Orueta U., Buiza, C. y Yanguas, J. (2010). Reserva cognitiva: evidencias, limitaciones y líneas de investigación futura. Revista Española de Geriatría y Gerontología, 45, 150-155.

Dixon, R. A. y de Frias, C. M. (2004). The Victoria Longitudinal Study: From characterizing cognitive aging to illustrating changes in memory compensation. Aging, Neuropsychology, and Cognition, 11, 346-376.

Dixon, R. A., de Frias, C. M. y Bäckman, L. (2001). Characteristics of selfreported memory compensation in older adults. Journal of Clinical and Experimental Neuropsychology, 23, 650-661.

Dixon, R. A. y Hultsch, D. F. (1983). Structure and development of metamemory in adulthood. Journal of Gerontology, 38, 682-688.

Dixon, R. A., Gagnon, L. M. y Crow, C. B. (1998). Collaborative memory accuracy and distortion: Performance and beliefs. En M. J. Intons-Peterson y D. L. Best (Eds.), Memory distortions and their prevention (pp. 63-88). Mahwah, NJ: Erlbaum.

Drag, L. L. y Bieliauskas, L. A. (2010). Contemporary review 2009: Cognitive aging. Journal of Geriatric Psychiatry and Neurology, 23, 75-93.

Folstein, M., Folstein, S. y Mc Hugh, P. (1975). Mini Mental State. A practical method for grading the cognitive state of patients for the clinical. Journal of Psychiatric Research, 12, 189-198.

Frias, C. M., Dixon, R. A. y Bäckman, L. (2003). Use of memory compensation strategies is related to psychosocial and health indicators. Journals of Gerontology Series B: Psychological Sciences and Social Sciences, 58, 12-22. tivo con el tiempo, en parte, debido a que aplican estrategias alternativas y eficaces para la realización de tareas cuando los métodos que empleaban anteriormente ya no son válidos. En este sentido, la capacidad de adaptación y aplicación de estrategias alternativas por parte de los sujetos con ARC, daría coherencia a su menor percepción de cambio en las funciones cognitivas, así como a la menor necesidad de aplicar esfuerzos compensatorios que aquellos sujetos con menor RC. Por otra parte, en este trabajo no se han observado diferencias en la aplicación de mecanismos de acomodación (éxito), de manera que ambos grupos desarrollan criterios de éxito inferiores a su capacidad para proteger el sentido de control al mantener objetivos más acordes con los recursos personales cognitivos y las demandas cotidianas de memoria.

Finalmente, hay una serie de limitaciones en este trabajo. Algunos efectos predictivos señalados en estudios previos no han podido ser observados aquí debido al tipo de diseño y muestreo. Otra posible limitación y futura vía de trabajo, hace referencia a la edad de los sujetos, dado que los problemas de memoria aumentan significativamente con la edad, futuros trabajos podrían reclutar una mayor proporción de adultos mayores en rangos de edad más altos (por ejemplo, 85 años) lo cual, ayudaría a asegurar que la compensación y RC se examinan en un punto en el que la pérdida de memoria es más probable que ocurra.

Frias, C. M. y Dixon, R. A. (2005). Confirmatory factor structure and measurement invariance of the Memory Compensation Questionnaire. Psychological Assessment, 17, 168-178.

Katzman, R. (1993). Education and the prevalence of dementia and Alzheimer's disease. Neurology, 43, 13-20.

León, I., García, J. y Roldán-Tapia, L. (2011). Construcción de la escala de reserva cognitiva en población española: estudio piloto. Revista de Neurología, 52, 653-660.

Lobo, A., Saz, P. y Marcos, G. (2002). Adaptación del Examen Cognoscitivo MiniMetal. Madrid: Tea Ediciones.

Lojo-Seoane, C., Facal, D. y Juncos-Rabadán, O. (2012). ¿Previene la actividad intelectual el deterioro cognitivo? Relaciones entre reserva cognitiva y deterioro cognitivo ligero. Revista Española de Geriatría y Gerontología, 47, 270-278.

Manly, J. J., Schupf, N., Tang, M. X., Weissm, C. C. y Stern. Y. (2007). Literacy and cognitive decline among ethnically diverse elders. En Y. Stern (Ed.), Cognitive reserve theory and applications (pp. 219-236). New York: Taylor \& Francis.

Meléndez, J. C., Tomás, J. M. y Navarro, E. (2007). Análisis de las redes sociales en la vejez a través de la entrevista Manhein de apoyo social. Salud Pública de México, 49, 408-416.

Meléndez, J. C., Mayordomo, T. y Sales, A. (2012). Comparación entre ancianos sanos con alta y baja reserva cognitiva y ancianos con deterioro cognitivo. Universitas Psychologica, 12, 73-80.

Meléndez, J. C., Mayordomo, T., Sales, A., Cantero, M. J. y Viguer, P. (2013). How we compensate for memory loss in old age: Adapting and validating the Memory Compensation Questionnaire (MCQ) for Spanish populations. Archives of Gerontology and Geriatrics, 56, 32-37.

Mortimer, J. A., Schuman, L. y French, L. (1981). Epidemiology of dementing illness. In J. A. Mortimer, \& L. M. Schuman (Eds.), The epidemiology of dementia: Monographs in epidemiology and biostatistics (pp. 323-333). New York: Oxford University Press.

Moscovitch, M. (1982). A neurological approach to perception and memory in normal and pathological aging. En F.I.M. Craik y S. Trehub (Eds.), Aging and cognitive processes (pp. 55-79). New York: Plenum Press.

Rami, L., Valls-Pedret, C., Bartrés-Faz, D., Caprile, C., Solé-Padullés, C., Castellví, M., ..., y Molinuevo, J. L. (2011). Cuestionario de reserva cognitiva. 
Valores obtenidos en población anciana sana y con enfermedad de Alzheimer. Revista de Neurología, 52, 195-201.

Santamarina Pérez, P. y Corral, M. (2009). Influencia de la reserva cognitiva en el rendimiento neuropsicológico de los pacientes con epilepsia. Medicina Clínica, 132, 459-462.

Scarmeas, N. (2007). Life style patterns and cognitive reserve. En Y. Stern (Ed.), Cognitive reserve: Theory and applications (pp. 187-206). New York: Taylor \& Francis.

Scarmeas, N., Zarahn, E., Anderson, K. E., Habeck, C., Hilton, J., Flynn, J., ..., y Stern, Y. (2003). Association of life activities with cerebral blood flow in Alzheimer's disease: implications for the cognitive reserve hypothesis. Archives of Neurology, 60, 359-365.

Solé-Padullés, C., Bartrés-Faz, D., Junqué, C., Vendrell, P., Rami, L., Clemente, I.,..., y Molinuevo, J. L. (2009). Brain structure and function related to cognitive reserve variables in normal aging, mild cognitive impairment and Alzheimer's disease. Neurobiology of Aging, 30, 1114-1124.

Stern, Y. (2002). What is cognitive reserve? Theory and research application of the reserve concept. Journal of the International Neuropsychological Society, 8, 448460.

Stern, Y. (2003). The concept of cognitive reserve. A catalyst for research. Journal of Clinical and Experimental Neuropsychology, 25, 589-593.
Stern, Y. (2006). Cognitive reserve and Alzheimer disease. Alz̧heimer Disease and Associated Disorders, 20, 112-117.

Stern, Y. (2009). Cognitive reserve. Neuropsychologia, 47, 2015-2028.

Stern, Y., Habeck, C., Moeller, J., Scarmeas, N., Anderson, K. E., Hilton, H. J., ... y Heertum, R. (2005). Brain networks associated with cognitive reserve in healthy young and old adults. Cerebral Cortex, 15, 394-402.

Valenzuela, M. y Sachdev, P. (2006a). Brain reserve and dementia: A systematic review. Psychological Medicine, 36, 441-454.

Valenzuela, M. y Sachdev, P. (2006b). Brain reserve and cognitive decline: A nonparametric systematic review. Psychological Medicine, 36, 1065-1073.

Van der Elst, W., Hoogenhout, E., Dixon, R., De Groot, R. y Jolles, J. (2011). The Dutch Memory Compensation Questionnaire: Psychometric properties and regression-based norms. Assessment, 18, 517-529.

Wechsler, D. (2001). Wechsler Intelligence Scale for Adults-III (WAIS-III). Madrid: TEA ediciones.

Wilson, B. A. (1999). Memory rehabilitation in brain injured people. En D. T. Stuss, G. Winocur y I. H. Robertson (Eds.), Cognitive neurorehabilitation (pp. 333-346). Cambridge: Cambridge University Press.

(Articulo recibido: 21-1-2013; revisión recibida: 6-11-2013; aceptado: 8-4-2014) 